$\xi=-1$

\title{
Implementing an educational program to increase preoperative screening for obstructive sleep apnea using the stop-bang questionnaire
}

\author{
Adam Davies *, W. Patrick Monaghan, Gerard T. Hogan \\ University of North Florida Brooks College of Health Jacksonville, Florida 32224 \\ *Corresponding author E-mail:
}

\begin{abstract}
Background: Obstructive sleep apnea (OSA) is a potentially fatal disease process that has been linked to higher rates of morbidity and mortality as well as increased perioperative complications. OSA is characterized by repetitive pauses in breathing during sleep. Greater than $92 \%$ of women and $82 \%$ of men who are plagued by moderate to severe sleep apnea are undiagnosed and may go unrecognized in the perioperative setting. The gap between a high prevalence of undiagnosed OSA in the adult population and the low level of clinical recognition has been well-documented. The term "STOP-BANG" is an acronym for eight independent elements predictive of OSAthree are OSA-related symptoms, three are physiological measurements, and two are patient characteristics.

Methods: This project used a quasi-experimental design using a 16-question self-developed survey based on the technology acceptance model (TAM). Participants were asked to read an educational pamphlet on OSA and then complete the survey.

Results: This study found strong evidence to suggest that among Certified Registered Nurse Anesthetists (CRNAs) and Student Registered Nurse Anesthetists (SRNAs), those with higher scores on Perceived Ease of Use (PEOU), Perceived Usefulness (PU), and Attitude toward Use (AT), tend to have a higher Behavioral Intention to Use (BIU) the STOP-BANG screening tool.

Conclusions: The results suggest that programs targeted at raising CRNAs' and SRNAs' PEOU, PU, and AT regarding the STOPBANG questionnaire will culminate in increased use of the STOP-BANG screening tool. The use of this screening tool will detect patients previously unidentified as having OSA, and ultimately prevent perioperative complications associated with this disease.
\end{abstract}

Keywords: Obstructive Sleep Apnea (OSA); STOP-BANG Questionnaire; Preoperative Screening; Anesthesia Complications; Technology acceptance model (TAM).

\section{Introduction}

Obstructive sleep apnea (OSA) is a chronic disease characterized by repetitive pauses in breathing during sleep that can lead to hypoxia and hypercapnia. OSA is defined by a pause in breathing that lasts at least 10 seconds and a decrease in oxygen saturation by $4 \%$ from the patient's baseline oxygen saturation (Henrichs \& Walsh, 2012; Mehta et al., 2014; Singh et al., 2013). The obstructive breathing pattern characteristic of OSA occurs when the muscles that typically support an open airway during sleep, relax in tone causing the airway to collapse and airflow to decrease or cease altogether (Henrichs \& Walsh, 2012).

OSA is a potentially fatal disease process and has been independently associated with higher rates of morbidity and mortality as well as increased perioperative complications, including a higher incidence of difficult intubation, obstructive breathing, postoperative complications, and increased length of hospital stays (Mehta et al., 2014; Singh et al., 2013). A recent meta-analysis found that OSA was associated with a higher risk of postoperative cardiac events, respiratory failure, and intensive care unit (ICU) admission (Kaw et al., 2012). Complications that may arise in the perioperative period for patients with OSA include new-onset arrhythmias, heart failure, hypotension, hypertension, myocardial ischemia or infarction, cerebrovascular stroke, disorientation, hypoxemia, atelectasis, pulmonary embolism, and pneumonia.
(Corso et al., 2014; Kulkarni, Horst, Eberhardt, Kumar, \& Sarker, 2014; Vasu et al., 2010).

It is believed perioperative complications are exacerbated in patients with OSA due to a variety of reasons. Patients with OSA experience a greater sensitivity to anesthetic agents, particularly opioids, and the added central nervous system depression brought on by these agents further promotes sleep disordered breathing through depression of ventilatory drive. Anesthetics and analgesics also alter upper airway tone, making it more prone to collapse and obstruction. Additionally, rapid eye movement (REM) sleep is greatly diminished if not completely absent the first night following surgery and then rebounds in the following 48 hours, subjecting the patient to increased hypoxemic obstructive episodes during this period (Vasu et al., 2010). Patients with moderate-to-severe OSA experience an adjusted hazard ratio for all-cause mortality that is three to six times higher than those without OSA (Singh et al., 2013).

OSA prevalence ranges from $9 \%$ to $24 \%$ in the general population based on numerous epidemiological studies (Singh et al., 2013). It is estimated that more than 18 million Americans suffer from OSA. More than $92 \%$ of women and $82 \%$ of men who suffer from moderate to severe sleep apnea are undiagnosed (Henrichs \& Walsh, 2012; Mehta et al., 2014; Singh et al., 2013). The prevalence of OSA in surgical candidates is higher than the general population, and varies by surgery type, with the highest prevalence in patients presenting for bariatric surgery (Chung et al., 
2013; Singh et al., 2013). The occurrence of OSA is expected to increase as the incidence of obesity escalates and the populations' life expectancy grows (Henrichs \& Walsh, 2012; Qaseem et al., 2014).

The gap between a high prevalence of undiagnosed OSA in the population and the low level of clinical recognition has been a recognized and well-documented issue (Singh et al., 2012). The diagnosis of OSA requires polysomnography (PSG), which involves time-consuming and expensive overnight monitoring at a limited number of testing centers (Henrichs \& Walsh, 2012; Mehta et al., 2014; Singh et al., 2013). It would be demanding and costly to implement prescribed testing for a large population. This deterrent has led to the development of validated questionnaires that use data from known risk factors for OSA and from polysomnography data.

\section{Literature review}

Numerous studies and clinical reviews have examined the efficacy of using the STOP-BANG questionnaire to screen for OSA (Chung et al., 2012; Chung et al., 2013; Dolezal, Cullen, Harp, \& Mueller, 2011; Farney, Walker, Farney, Snow, \& Walker, 2011; Gali, Whalen, Schroeder, Gay, \& Plevak, 2009; Ha, Lee, Abdullah, \& van Hasselt, 2014; Luo, Huang, Zhong, Xiao, \& Zhou, 2014; Silva, Vana, Goodwin, Sherrill, \& Quan., 2011; Vana, Silva, \& Goldberg, 2013). The STOP-BANG questionnaire was designed and validated to screen patients for undiagnosed OSA in the preoperative setting (Young, Peppard, \& Gottlieb, 2002). The term "STOP-BANG" is an acronym for eight independent elements predictive of OSA - three are OSA-related symptoms (snoring, tiredness, and observed periods of apnea), three are physiological measurements (body mass index, neck circumference, and hypertension), and two are patient characteristics (age and gender) (Singh et al., 2013). The score is created using yes/no answers, carrying a score of $1 / 0$ respectively. A score of 3 or higher has been shown to have a sensitivity of $93 \%$ for detecting mild OSA and a score of 5 to 8 a $100 \%$ sensitivity for moderate and severe OSA (Chung et al., 2012; Chung et al., 2013). The consensus of these studies is that the STOP-BANG questionnaire is a valid and reliable tool for assessing OSA risk in surgical and obese patients and is a practical, economical option to traditional overnight polysomnography.

The American Society of Anesthesiologists (ASA) addressed OSA in 2006 with practice guidelines which included assessment of patients for possible OSA before surgery and careful postoperative monitoring for those suspected to be at high risk. The ASA guidelines also included recommendations to evaluate patients who may be at high risk based on clinical suspicion preoperatively (Gross et al., 2006).

\section{Study design \& methods}

This project used a quasi-experimental design using a 16-question self-developed survey based on the technology acceptance model (TAM).

Perceived Ease of Use (PEOU), Perceived Usefulness (PU), and Attitude toward Use (AT) were independent variables of this study measured on a continuous measurement scale with a range of 1 to 5. Each value was derived from three specific survey questions. Response choices to the survey questions were coded as $1=$ Strongly Disagree; 2 = Disagree; 3 = Neither Agree nor Disagree; $4=$ Agree, and; $5=$ Strongly Agree. Behavioral Intention to Use (BIU) was the dependent variable evaluated in this study and was again measured from three specific questions. Again these questions were constructed with a response measurement scale ranging from 1 to 5 ( $1=$ Strongly Disagree; $5=$ Strongly Agree $)$. Thus, smaller scores indicated less intention to use the STOP-BANG questionnaire in clinical practice while larger scores indicated greater intention to utilize STOP-BANG screening.
An educational fact sheet highlighting the prevalence of undiagnosed OSA, complications from OSA, utilization of the STOPBANG questionnaire to screen for OSA, and anesthetic management of patients with OSA was constructed from articles found during the literature review. After appropriate Internal Review Board (IRB) approval, a link to Qualtrics online survey tool was provided to the Florida Association of Nurse Anesthetists (FANA) to distribute to all CRNAs and SRNAs who currently hold membership with FANA to maintain the anonymity of the participants. All participants were asked to provide consent before being allowed to view the educational pamphlet or survey questions. Once consent was obtained, participants were able to access and review the educational pamphlet. Participants were then allowed to access and complete the survey questions.

Research Hypothesis

H1. What, if any, correlation is there between behavioral intention to use (BIU) the STOP-BANG questionnaire and the perceived ease of use (PEOU) of the STOP-BANG among CRNAs and SRNAs?

$\mathrm{H} 2$. What, if any, correlation is there between behavioral intention to use (BIU) the STOP-BANG questionnaire and the perceived usefulness (PU) of the STOP-BANG among CRNAs and SRNAs? $\mathrm{H} 3$. What, if any, correlation is there between behavioral intention to use (BIU) the STOP-BANG questionnaire and the attitude toward using (AT) the STOP-BANG among CRNAs and SRNAs? H4. Which combination of PEOU, PU, and AT better predict BIU than any single independent variable alone among CRNAs and SRNAs?

\section{Results}

Descriptive statistics were generated using the Qualtrics (2015) software. The power calculations were performed using the PASS software (Hintze, 2008). Hypotheses 1 through 3 were tested using Spearman's correlation coefficient. Although there are no formulas for calculating the power of the Spearman's correlation coefficient, the Spearman's correlation coefficient is simply the Pearson's correlation coefficient applied to the transformed (i.e. ranked) data. Thus, the effect sizes reported below can be considered a close approximation.

According to Cohen (1988), small, medium, and large effect sizes for hypothesis tests about the Pearson correlation coefficient (r) are: $r=0.1, r=0.3$ and $r=0.5$ respectively. A sample size of 150 produces $80 \%$ power to detect an effect size of 0.23 , which is a small to medium effect size. In this study, the effect sizes for hypotheses 1-3 ranged from 0.48 to 0.67 . Thus, the sample size was more than adequate for detecting meaningful effect sizes (medium or larger) for this study.

Hypothesis 4 was tested using stepwise multiple linear regression analysis. Power analysis for multiple linear regression analysis is based on the amount of change in R-squared $\left(\mathrm{r}^{2}\right)$ attributed to the variables of interest. According to Cohen (1988), small, medium, and large effect sizes for hypothesis tests about $\mathrm{r}^{2}$ are: $\mathrm{r}^{2}=0.0196$, $r^{2}=0.13$ and $r^{2}=0.26$ respectively. A sample size of 150 achieves $80 \%$ power to detect an $\mathrm{r}^{2}$ of 0.062 , which is a small effect size attributed to two independent variables (e.g. PU and AT) using an F-Test with a significance level (alpha) of 0.05. The observed effect size for Hypothesis 4 was 0.53 . Thus, a sample size of 150 was more than adequate to detect the observed effect sizes.

Among the 196 respondents to the study invitation, 40 (20.4\%) failed to answer all of the survey questions and were omitted from the analysis. Table 1 shows descriptive statistics for the independent and dependent variables. Considering the smallest possible score for each variable was 1.0 and the maximum possible score was 5.0, on average all of the scores were well above the midpoint of 3.0, with averages ranging from 4.11 (BIU) to 4.35 (PU). Thus, on average, study participants perceived the STOP-BANG to be a useful tool that is easy to use, and demonstrated a positive attitude toward using it, and had a high level of intention to use the STOP-BANG in the future. Nevertheless, all variables showed 
significant variation with scores ranging from 1.0 to 5.0 for the PEOU, and BIU, for example. So, at least some participants did not perceive the STOP-BANG to be easy to use, perceived a low level of usefulness, had a negative attitude toward using it, and had a low level of behavioral intention to use.

Table 1: Descriptive Statistics for the Independent and Dependent Variables

\begin{tabular}{|c|c|c|c|c|}
\hline & $\begin{array}{l}\mathrm{N} \\
\text { ValidMissing }\end{array}$ & Mean $\begin{array}{l}\text { Std. Devia- } \\
\text { tion }\end{array}$ & Minir & nMaximum \\
\hline $\begin{array}{l}\text { Perceived Ease of } \\
\text { Use }^{\text {a }}\end{array}$ & 1500 & 4.3111 .65158 & 1.00 & 5.00 \\
\hline $\begin{array}{l}\text { Perceived Usefulness } \\
\text { a }\end{array}$ & $150 \quad 0$ & 4.3511 .58922 & 1.33 & 5.00 \\
\hline $\begin{array}{l}\text { Attitude Toward } \\
\text { Using }^{\text {a }}\end{array}$ & 1500 & 4.2222 .65728 & 2.00 & 5.00 \\
\hline $\begin{array}{l}\text { Behavioral Intention } \\
\text { to Use }{ }^{\mathrm{b}}\end{array}$ & 1500 & 4.1133 .80715 & 1.00 & 5.00 \\
\hline
\end{tabular}

a. Independent Variable

b. Dependent Variable

This study found strong evidence to suggest that all three independent variables were positively correlated with the dependent variable. That is to say, among CRNAs and SRNAs, those with a higher score on PEOU, PU, and AT, tend to have a higher intention to use (BIU) the STOP-BANG screening tool. The results suggest that effective implementation of the STOP-BANG questionnaire may require that CRNAs and SRNAs have a high level of PEOU, PU, and AT, in an effort to increase their level of BIU. Thus, if interventions such as the informational pamphlet utilized in this study can be developed to increase PEOU, PU, and AT, this may increase BIU, which would be expected to increase actual use of the STOP-BANG, which would be expected to decrease perioperative complications.

\section{Discussion}

As previously stated in the literature review, the STOP-BANG questionnaire is a useful tool for predicting OSA risk and has been validated numerous times for use in screening of surgical patients preoperatively for OSA. The literature review also revealed that many patients with OSA are undiagnosed and that wide-scale polysomnography testing is costly, time-consuming, and impractical. The STOP-BANG questionnaire is practical, simple to use, easily interpreted, and an economical option to traditional overnight polysomnography and can be implemented successfully on a wide scale basis in the preoperative setting. While it is known that the STOP-BANG questionnaire is an exemplary screening tool, CRNAs and SRNAs PEOU, PU, AT, and BIU were unknown until this study.

This study found strong evidence to positively correlate PEOU, PU, and AT to BIU. CRNAs and SRNAs who ranked the STOPBANG questionnaire higher in PEOU, PU, and AT scoring had a higher intent to utilize the STOP-BANG questionnaire in their respective clinical practices. The results of this study suggest that a positive trending of perceptions and attitudes toward the STOPBANG questionnaire are needed in order to increase the provider's intent to use this OSA screening tool. An effort to increase preoperative STOP-BANG screening would require CRNAs and SRNAs having high perceived ease of use, perceived usefulness, and positive attitudes toward using the STOP-BANG questionnaire in order to translate these attitudes into action. This study showed that interventions such as the educational pamphlet can be formulated to increase PEOU, PU, and AT, as this may subsequently increase BIU ultimately leading to increased preoperative screening and decreased perioperative complications.

\section{Limitations}

The first limitation is that the self-developed survey based on the TAM model was not validated prior to utilization. A validated survey may have yielded high quality data with greater comparability, although a validated tool on this specific topic of perioperative OSA screening does not exist to the author's knowledge. The second limitation of this study is its relatively small sample size in relation to the total number of CRNAs and SRNAs globally. As stated previously the sample size of 150 produced $80 \%$ power to detect an effect size of 0.23 , which is a small to medium effect size. In this study, the effect sizes for hypotheses 1-3 ranged from 0.48 to 0.67 . Thus, the sample size was more than adequate for detecting meaningful effect sizes (medium or larger) for this study. It is difficult however for the results of this study to be generalized to the entire population of CRNAs and SRNAs due to the small sample size.

\section{Conclusion}

The underlying purpose of this quality improvement project was to increase screening and ultimately reduce perioperative complications by implementing an educational program targeted towards CRNAs and SRNAs to highlight the significance of the STOPBANG questionnaire in evaluating patients for OSA. This study found strong evidence to suggest that among CRNAs and SRNAs, those with higher scores on PEOU, PU, and AT, tend to have a higher intention to use (BIU) the STOP-BANG screening tool. The results suggest that programs targeted at raising CRNAs' and SRNAs' PEOU, PU, and AT regarding the STOP-BANG questionnaire will culminate in increased use of the STOP-BANG screening tool, detecting patients previously unidentified as having OSA, and ultimately preventing perioperative complications associated with this disease.

\section{References}

[1] Cohen, J. (1988). Statistical Power Analysis for the Behavioral Science. New Jersey, Lawrence Erlbaum Associates, Inc. Lawrence Erlbaum Associates, Inc.; 365 Broadway; Hillsdale, New Jersey 07642

[2] Chung, F., Subramanyam, R., Liao, P., Sasaki, E., Shapiro, C., \& Sun, Y. (2012). High STOP-BANG score indicates a high probability of obstructive sleep apnoea. British Journal of Anaesthesia, 108, 768-775. http://dx.doi.org/10.1093/bja/aes022.

[3] Chung, F., Yang, Y., \& Liao, P. (2013). Predictive performance of the STOP-BANG score for identifying obstructive sleep apnea in obese patients. Obesity Surgery, 23, 2050-2057. http://dx.doi.org/10.1007/s11695-013-1006-z.

[4] Dolezal, D., Cullen, L., Harp, J., \& Mueller, T. (2011). Implementing preoperative screening of undiagnosed obstructive sleep apnea. Journal of Perianesthesia Nursing, 26, 338-342. http://dx.doi.org/10.1016/j.jopan.2011.07.003.

[5] Farney, R. J., Walker, B., Farney, R. M., Snow, G., \& Walker, J. (2011). The STOP-BANG equivalent model and prediction of severity of obstructive sleep apnea: Relation to polysomnnographic measurements of the apnea-hypopnea index. Journal of Clinical Sleep Medicine, 7, 459-465. http://dx.doi.org/10.5664/jcsm.1306.

[6] Gali, B., Whalen, F., Schroeder, D., Gay, P., \& Plevak, D. (2009). Identification of patients at risk for postoperative respiratory complications using a preoperative obstructive sleep apnea screening tool and postanesthesia care assessment. Anesthesiology, 110, 869877. http://dx.doi.org/10.1097/ALN.0b013e31819b5d70.

[7] Gross, J., Bachenberg, K., Benumof, J., Caplan, R., Connis, R., Cote, C., Yu, S. (2006). Practice guidelines for the perioperative management of patients with obstructive sleep apnea: A report by the American Society of Anesthesiologists task force on perioperative management of patients with obstructive sleep apnea. Anesthesiology, 104, 1081-1093. Retrieved from http://journals.lww.com/Anesthesiology http://dx.doi.org/10.1097/00000542-200605000-00026

[8] Ha, S., Lee, D., Abdullah, V., \& van Hasselt, C. (2014). Evaluation and validation of four translated Chinese questionnaires for obstructive sleep apnea patients in Hong Kong. Sleep and Breathing. Retrieved from http://link.springer.com/journal/11325 http://dx.doi.org/10.1007/s11325-013-0889-1. 
[9] Henrichs, B., \& Walsh, R., (2012). Is that snoring something to worry about? Anesthetic implications for obstructive sleep apnea. AANA Journal, 80, 393-401. Retrieved from http://www.aana.com/

[10] Hintze, J. (2008). PASS 2008 [Computer Software]. Kaysville, UT: NCSS, LLC.

[11] Kaw, R., Chung, F., Pasuleti, J., Mehta, J., Gay, P., \& Hernandez, A. (2012). Meta-analysis of the association between obstructive sleep apnoea and postoperative outcome. British Journal of Anesthesia, 109, 897-906. doi:10.1093/bja/aes308 http://dx.doi.org/10.1093/bja/aes308.

[12] Kulkarni, G. V., Horst, A., Eberhardt, J. M., Kumar, S., \& Sarker, S. (2014). Obstructive sleep apnea in general surgery patients: Is it more common than we think? American Journal of Surgery, 207(3), 436-40. http://dx.doi.org/10.1016/j.amjsurg.2013.09.018.

[13] Luo, J., Huang, R., Zhong, X., Xiao, Y., \& Zhou, J. (2014). STOPBANG questionnaire is superior to Epworth sleepiness scales, Berlin questionnaire, and STOP questionnaire in screening obstructive sleep apnea hypopnea syndrome patients. Chinese Medical Journal, 127, 3065-3070. Retrieved from http://www.cmj.org/ch/index.aspx.

[14] Mehta, P., Kochhar, G., Kalra, S., Maurer, W., Tetzlaff, J., Singh, G., Vargo, J. (2014). Can a validated sleep apnea scoring system predict cardiopulmonary events using propofol sedation for routine EGD or colonoscopy? A prospective cohort study. Gastrointestinal Endoscopy, 79, 436-444. http://dx.doi.org/10.1016/j.gie.2013.09.022.

[15] Qaseem, A., Dallas, P., Owens, D., Starkey, M., Holty, J., \& Shekelle, P. (2014). Diagnosis of obstructive sleep apnea in adults: A clinical practice guideline from the American College of Physicians. Annals of Internal Medicine, 16c, 210-220. http://dx.doi.org/10.7326/M12-3187.

[16] Qualtrics. (2015). Qualtrics [Computer Software]. Provo, UT: Qualtrics, LLC.

[17] Silva, G. E., Vana, K. D., Goodwin, J. L., Sherrill, D. L., \& Quan, S. F. (2011). Identification of patients with sleep disordered breathing: Comparing the four-variable screening tool, STOP, STOPBANG, and epworth sleepiness scales. Journal of Clinical Sleep Medicine, 7, 467-472. http://dx.doi.org/10.5664/jcsm.1308.

[18] Singh, M., Liao, P., Kobah, S., Wijeysundera, D., N., Shapiro, C. $\&$ Chung, F. (2013). Proportion of surgical patients with undiagnosed obstructive sleep apnoea. BJA: The British Journal of Anaesthesia, 110, 629-636. http://dx.doi.org/10.1093/bja/aes465.

[19] Vana, K., Silva, G., \& Goldberg, R. (2013). Predictive abilities of the STOP-BANG and Epworth sleepiness scale in identifying sleep clinic patients at high risk for obstructive sleep apnea. Research in $\begin{array}{llll}\text { Nursing Health, } & \text { 36(1), 84-94. }\end{array}$ http://dx.doi.org/10.1002/nur.21512.

[20] Vasu, T. S., Doghramji, K., Cavallazzi, R., Grewal, R., Hirani, A., Leiby, B., Witkowski, T. (2010). Obstructive sleep apnea syndrome and postoperative complications: Clinical use of the STOP-BANG questionnaire. Archives of Otolaryngology_Head \& Neck Surgery 136(10), 1020-1024. http://dx.doi.org/10.1001/archoto.2010.1020.

[21] Young, T., Peppard, P., \& Gottlieb, D. (2002). Epidemiology of obstructive sleep apnea: A population health perspective. American Journal of Respiratory \& Critical Care Medicine, 165, 1217-1239. Retrieved from http://www.atsjournals.org/journal/ajrccm. http://dx.doi.org/10.1164/rcem.2109080. 